\title{
Theoretical and Experimental Study on Longitudinal Impact of Tapered Rods
}

\author{
L. R. Hettche* \\ Institute for Basic Standards, National Bureau of Standards, Washington, D.C. 20234
}

(July 1, 1968)

\begin{abstract}
To examine the adequacy of the one-dimensional, rate-independent theory of plastic-wave propagation for annealed, commerically pure aluminum, experimental results from the longitudinal impact of uniform and tapered rods are compared to a theoretical analysis. The theoretical description is a numerical solution which utilizes the characteristic properties of the governing equations to construct difference relations for a constant mesh spacing. Numerical evidence of convergence and stability of the solution is presented. A constant-velocity boundary condition is defined by the axial collision of identical specimens, 6 inches $(152 \mathrm{~mm})$ in length and tapered slope varying between \pm 0.03 , using a $3 / 4$-inch $(19 \mathrm{~mm})$ bore gas gun. Strain-time profiles are measured at cross-section distances of 1,2 , and 4 inches $(25,51$, and $102 \mathrm{~mm})$ from the impact face with resistance gages. Quantitative agreement between theoretical prediction and experimental data, e.g., dispersive features and unloading patterns of the strain wave, show the theory and method of solution fo be a reasonably accurate model of the deformation. The selection of the specimen geometry as the experimentally controllable parameter in the longitudinal impact experiment is found to be a useful testing method in investigating the dynamic response of materials.
\end{abstract}

Key Words: Aluminum; experiments; longitudinal impact; numerical solution; one-dimensional; plastic-wave propagation; rate-independent theory; tapered rods.

\section{Introduction}

The one-dimensional, rate-independent theory of plastic-wave propagation in thin, uniform rods has been extensively studied and the object of experimental verification since its inception in the independent works of von Kármán [1] ${ }^{1}$, Taylor [2], and Rakhmatulin [3]. Assuming that the motion and stress state are uniaxial and that the stress-strain curve is not only strain-rate independent but also concave toward the strain axis, this theory predicts the following results: A strain of $\epsilon$ propagates along the bar with a speed $\lambda=\left[f^{\prime}(\epsilon) / \rho\right]^{1 / 2}$ where $f^{\prime}(\epsilon)$ is the slope of the stress-strain curve at the strain $\epsilon$, and $\rho$ is the mass density; the relationship between impact velocity $v$ and strain is given by $v=\int_{0}^{\epsilon} \lambda d \epsilon$. Experimental investigations have centered, for the most part, on verifying the above results and the strain-rate independent assumption of the stress-strain curve.

Conventionally, strain-rate independence of mechanical response is taken to imply that a material

\footnotetext{
*Present address: Naval Research Laboratory, Washington, D.C. 20390

${ }^{1}$ Figures in brackets indicate the literature references on page 239.
}

is characterized by an invariant stress-strain relation for increasing or constant values of strain (loading) and another stress-strain relation, usually linear, for decreasing values of strain (unloading). The separation of material response into loading and unloading is suggested by the well-known yielding phenomenon and elastic-plastic response of a uniaxial tension/ compression specimen. When abrupt yielding or unloading occurs under dynamic conditions, the discontinuous behavior of $f^{\prime}$ causes an elastic-plastic boundary to propagate in the solution field. This boundary not only separates regions of elastic and plastic resporise but also defines a locus of discontinuities in particle acceleration and propagation speed $\lambda$. Such a boundary is called a weak discontinuity in contrast to a strong discontinuity (shock) where stress and particle velocity are discontinuous. The location of the elastic-plastic boundaries is a domonant feature of the dynamic analysis and often necessitates a hybrid solution where analytic, graphical, and numerical techniques are employed. The analysis by Lee [4] involving the constant-velocity impact of a uniform rod of finite length is a notable example. In many cases, idealization of boundary conditions and stress-strain behavior are made to facilitate the identification of the elastic and plastic regions 
in the $x, t$-plane.

The degree to which the rate of strain influences the stress-strain curve has remained an active concern, as well as a point of controversy, among investigators. As emphasized by Bell [5], the governing stress-strain curve need not be the static relationship to classify a material response as strain-rate independent. Indeed, from his finite-amplitude; plastic-wave measurements Bell [6] has correlated the governing stressstrain curve for annealed, commerically pure aluminum with a rate-independent, parabolic law. This relationship is applicable between strain rates of $10 \mathrm{sec}^{-1}$ to $10^{3} \mathrm{sec}^{-1}$ and diverges from the static curve for strains above 0.02. Contrary to the results of Bell, however, dynamic stress-strain curves of 0 tempered, commercially pure aluminum published by several authors $[7,8]$ show a strong dependence of mechanical response to rate of loading. These data are obtained for a range of strain rates, $10^{-3} \mathrm{sec}^{-1}$ to $10^{3} \mathrm{sec}^{-1}$, using several loading techniques (e.g., pressurized gas loaders, and split Hopkinson bar). In each case, loads and displacements of adjacent loading heads are simultaneously recorded under near constant strain rates. Implicit in such a measurement and a limitation to maximum loading rates obtainable in such devices is the assumption that a uniaxial strain is uniformly distributed throughout the specimen.

Within the general context of the above discussion and with the anticipation of developing a useful testing technique, the longitudinal impact of rods of nonuniform cross-sectional areas are examined in this study. The objective here is to define a constant and physically realizable loading (boundary) condition in the longitudinal impact problem, and through specimen geometry alter both the dispersive characteristics and unloading pattern of the deformation. In this way, experimental observations are readily made to test various aspects of theory and method of solution. Results are reported for three specimen geometries of annealed, commercially pure aluminum (1100-0). In each case, the boundary condition corresponds to a constant-velocity impact except that the step in velocity has a finite rise time.

The theoretical analysis is a numerical solution of the one-dimensional, rate-independent equations of plastic-wave propagation. The characteristic properties of the governing equations are utilized to construct difference relations for a constant mesh spacing. Boundaries separating regions of elastic and plastic response are located in the $x, t$-plane by an iterative scheme where the solution at each point is compared to its previous strain history. Shock-type motion is not encountered since only stress-strain curves concave toward the strain axis are considered. Numerical evidence of convergence and stability of the method is examined by several test problems.

A constant-velocity loading of 800 in per second (20 meters per second) is achieved by the axial collision of identical specimens. Strain-time profiles are measured on the stationary specimens at crosssection distances of 1,2 , and 4 in $(25,51$, and $102 \mathrm{~mm})$ from the impact face with resistance gages. In each test, the responses at two positions are recorded from a dual-beam oscilloscope, and at least three tests are made for each of the three specimen geometries considered.

\section{Numerical Solution}

The integration of hyperbolic differential equations in two independent variables by finite difference methods is a well developed area of applied mathematics. Chapter 2 of the text by Forsythe and $\mathbf{W} /$ asow [9] gives important theoretical results and surveys existing methods. The selection or development of any one scheme usually is influenced if not dictated by the problem in question. For the class of elasticplastic problems considered in this study, the governing equations are first transformed into the normal form (see appendix for derivation)

$$
\left(\frac{\partial \sigma}{\partial t} \pm \lambda \frac{\partial \sigma}{\partial x}\right) \pm \lambda \rho\left(\frac{\partial v}{\partial t} \pm \lambda \frac{\partial v}{\partial x}\right) \pm \lambda \frac{\partial A}{\partial x} \frac{\sigma}{A}=0
$$

where $\sigma$ is the nominal stress, $v$ is the particle velocity, $\rho$ the mass density, and $A$ the cross-sectional area. The coefficient $\lambda=\left(f^{\prime} / \rho\right)^{1 / 2}$ is identified as the characteristic wave speed and $f^{\prime}$, the slope of the stressstrain diagram, satisfies the following relations:

$$
\begin{gathered}
f^{\prime}=E \text { when } \epsilon<\epsilon_{y} \text { or } \dot{\epsilon}^{p}=0 \\
E>f^{\prime}>0, \text { and } f^{\prime \prime}<0 \text { when } \dot{\epsilon}^{p} \neq 0
\end{gathered}
$$

where $E$ is Young's modulus, $\epsilon_{y}$ is the strain at the proportional limit, and $\dot{\epsilon}^{p}$ is plastic-strain rate. In the above formulation compressive stresses and strains are positive, and the stress-strain behavior is restricted to linear elastic response on stress reversal.

A desirable property of hyperbolic equations transformed to the normal form is that the dependent variables are differentiated in a common direction. That is, $\sigma$ and $v$ are differentiated along the so-called characteristic curve $x=x(t)$ for which $d x / d t= \pm \lambda$. This form of the governing equations is amenable to finite difference approximation. The approach here is to write eq (1) as

$$
\begin{gathered}
d \sigma+\lambda \rho d v+\lambda \frac{\partial A}{\partial x} \frac{\sigma}{A} d t=0 ; \frac{d x}{d t}=\lambda \\
d \sigma-\lambda \rho d v-\lambda \frac{\partial A}{\partial x} \frac{\sigma}{A} d t=0 ; \frac{d x}{d t}=-\lambda
\end{gathered}
$$

where $d \sigma$ and $d v$ in eqs (3) and (4) are differentials along the + and - characteristic curves respectively.

To construct a difference equation problem to the above equations consider a rectangular mesh in the $x, t$-plane of constant spacing, $\Delta x$ and $\Delta t$, as shown in figure 1 . At the points $\mathrm{P}, \mathrm{Q}$, and $\mathrm{R}$ along a constant time line $t$, the solution is considered known. The solution is sought at point $\mathrm{S}$ at a later time $t+\Delta t$. Equations (3) and (4) can be integrated along the, as 


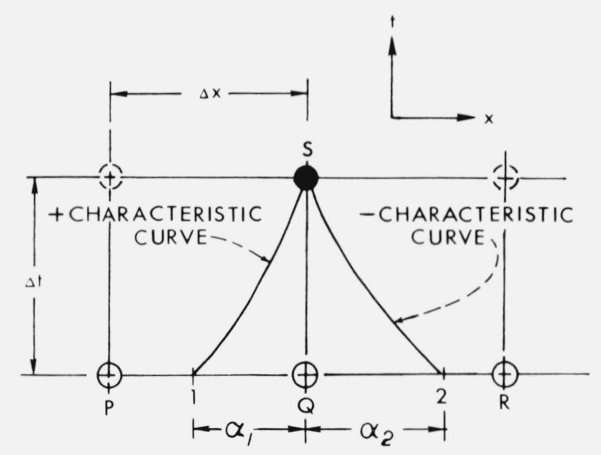

FIGURE 1. Characteristic curves at a typical mesh point.

yet unknown, + and - characteristic curves from their intersection with the line $t$, point 1 and 2 respectively, to point $\mathrm{S}$. Thus, along the + characteristic curve

$\sigma(\mathrm{S}) / \lambda(\mathrm{S})-\sigma(1) / \lambda(1)+\rho[v(\mathrm{~S})-v(1)]$

$$
-\int_{1}^{s} \sigma d \lambda^{-1}+\int_{1}^{s} \frac{\partial A}{\partial x} \frac{\sigma}{A} d t=0
$$

and along the - characteristic curve

$$
\begin{aligned}
\sigma(\mathrm{S}) / \lambda(\mathrm{S})-\sigma(2) / \lambda(2)-\rho[v(0)-v(2)] \\
-\int_{2}^{s} \sigma d \lambda^{-1}-\int_{2}^{s} \frac{\partial A}{\partial x} \frac{\sigma}{A} d t=0
\end{aligned}
$$

where the numbers in parentheses and limits of integration denote the value of the variables at the correspondingly numbered points in figure 1 .

Thus far, the solution of the quasi-linear, elasticplastic equations has been reduced to the evalation of the integrals in eqs (4) and (5), the location of the intersection of characteristic curves with the line $t$ (i.e., points 1 and 2), and obtaining the values of stress and velocity at the latter points. To ensure that the characteristic curves intersect between adjacent mesh points and to satisfy the important convergence requirement that the interval of dependence for the difference equations is greater than or equal to the one for the differential equations, the incremental distance $\Delta x$ to the incremental time $\Delta t$ ratio is taken as the elastic-propagation velocity. That is

$$
\frac{\Delta x}{\Delta t}=(E / \rho)^{1 / 2} .
$$

This result follows from the definition of the characteristic curves and relationships (2).

The distance $\alpha_{i}$, locating points 1 and 2 in figure 1 , is given by the line integral

$$
\alpha_{i}=\int_{i}^{s} \lambda d t ; \quad i=1,2
$$

along the respective curves. When $\lambda$ is a constant in the region of interest (the characteristic curves are linear), the integration of eq (8) is elementary and the first integrals in eqs (5) and (6) are seen to vanish. In the more general case, $\alpha_{i}$ is obtained from the difference approximation

$$
\alpha_{i}=\int \lambda d t=\bar{\lambda}_{i} \Delta t+0\left(h^{2}\right), \quad i=1,2
$$

where, for example,

$$
\bar{\lambda}_{2}=\lambda(\mathrm{S}) / 2+\left[\lambda(\mathrm{R}) \alpha_{2}+\lambda(\mathrm{Q})\left(\Delta x-\alpha_{2}\right)\right] / 2 \Delta x .
$$

Consistent with the approximation made in eq (9), the value of the dependent variables at points 1 and 2 are determined by linear interpolation between adjacent points, for example,

$$
\sigma(2)=\sigma(\mathrm{Q})+\frac{[\sigma(\mathrm{Q})-\sigma(\mathrm{R})] \alpha_{2}}{\Delta x} .
$$

To complete the difference approximations, the integrals of eqs (5) and (6) are approximated by the following relations:

$$
\begin{array}{r}
\int_{i}^{s} \sigma d \lambda^{-1}=[\sigma(\mathrm{S})+\sigma(i)] \frac{\lambda(i)-\lambda(\mathrm{S})}{2 \lambda(i) \lambda(\mathrm{S})}+0\left(h^{2}\right) \\
\int_{i}^{s} \frac{\partial A}{\partial x} \frac{\sigma}{A} d t=\left(\left.\frac{\partial A}{\partial x} \frac{\sigma}{A}\right|_{s}+\left.\frac{\partial A}{\partial x}\right|_{i}\right) \frac{\Delta t}{2}+0\left(h^{2}\right) .
\end{array}
$$

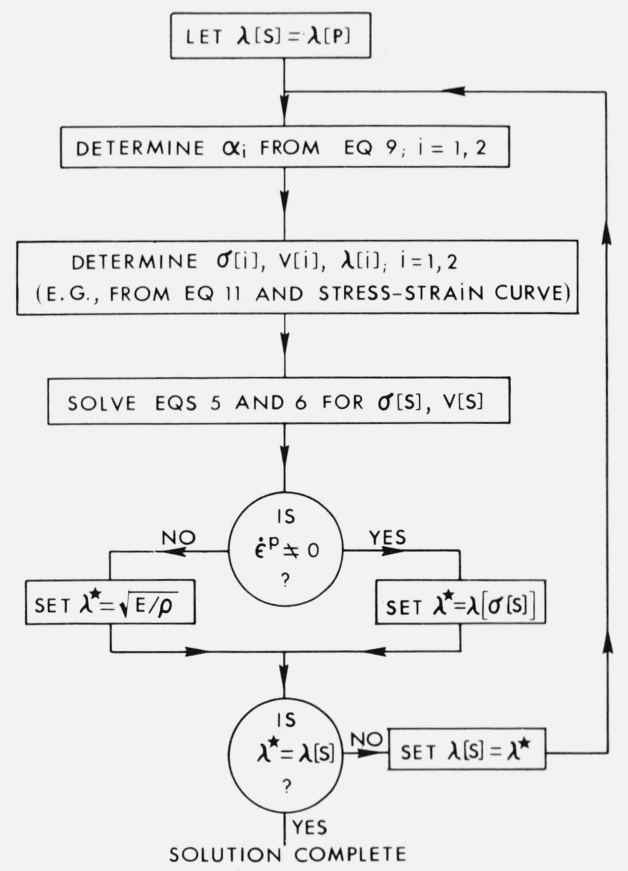

Figure 2. Flow diagram of numerical solution. 
The $0\left(h^{2}\right)$ approximation in the above relations hold if the integrands satisfy a Lipschitz condition.

With the given difference approximations, eqs (9) through (13), a noniterative solution of eqs (5), (6), and (8) can be obtained by approximating $\lambda(s)$ [e.g., $\lambda(s)=\lambda(\mathrm{Q})]$. This simplification however, proved unsatisfactory for the problems considered in this paper, and an iterative scheme was formulated. This solution for a typical mesh point, see figure 1 , is conveniently illustrated in the flow diagram of figure 2 . When adjacent points span an elastic-plastic boundary, the location of the boundary is taken at the midpoint of the mesh spacing. For example, if point $\mathrm{P}$ is unloaded and $\alpha_{1} \geqq 0.5 \Delta x, \lambda(1)=(E / \rho)^{1 / 2}$. Otherwise, $\lambda(1)=\lambda[\sigma(1)]$. Thus, the unloading boundary is located to within a mesh spacing in the numerical output and estimated in the calculation to within onehalf of a mesh spacing.

\section{Example of Numerical Solution}

To illustrate the behavior of the numerical solution, the plastic-wave motion in an infinite, uniform rod subject to a stress-pulse loading at the end is examined. This problem was chosen for illustration since it contains the general features of the unloading phenomena in elastic-plastic analyses and a sufficiently accurate graphical solution is available for comparison. The boundary condition is given by

$$
\sigma(0, t)=8 \sigma_{y} \frac{t}{T}(1-t / T)
$$

where $\sigma_{y}$ is the yield stress, $\sigma(0, t)$ is the stress at $x=0, t$ again denotes time, and $T$ is the pulse duration. The stress at the end of the rod, $x=0$, is seen to obtain a maximum value of $2 \sigma_{y}$ at $t=0.5 T$. The stress-strain curve is a bilinear relationship with an elastic modulus to plastic-tangent modulus ratio $\left(E / E^{\prime}\right)$ of 16 . The

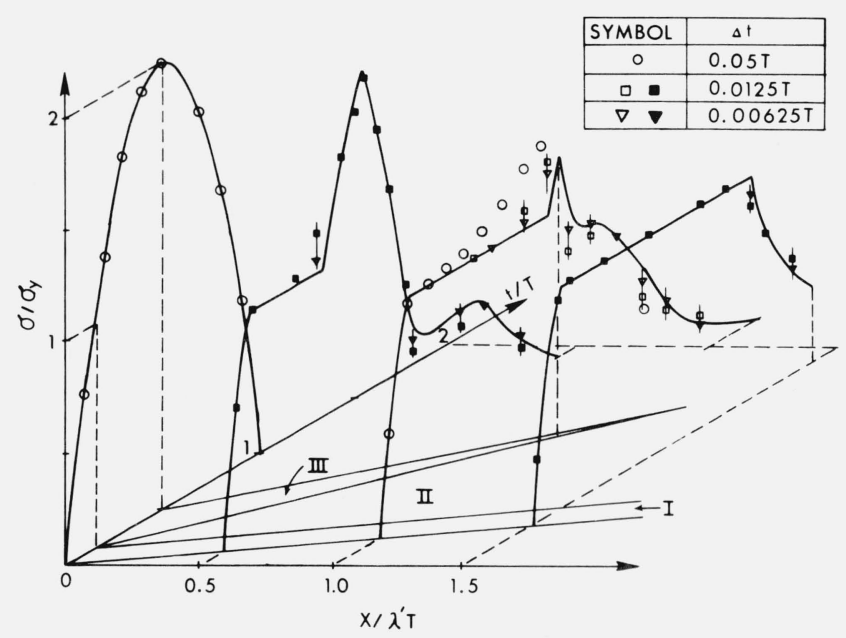

FIGURE 3. Graphical and numerical solution of stress pulse problem (dimensionless stress-time-x plot). elastic-wave speed is, therefore, 4 times the plasticwave speed $\left(\lambda=4 \lambda^{\prime}\right)$.

An overall picture of the deformation is contained in the dimensionless $x / \lambda^{\prime} T-t / T$ plane of figure 3 . The initial elastic response, region $\mathrm{I}$, is bounded by the parallel elastic-characteristic curves $x=\lambda t$. On yielding, $\sigma(0, t)=\sigma_{y}$, the diverging elastic-characteristic and plastic-characteristic curves define a constant state solution in region II. For $t$ greater than $0.5 T$, the stress boundary condition decreases in absolute value and an unloading boundary propagates into the solution field. The area between the unloading boundary and the initial plastic disturbance, region III, defines the zone of plastic deformation. Above the unloading boundary the response is elastic. The graphical solution of this problem can be found in the book by Rakhmatulin and Den' Yanov [10] and was originally published by Shapiro [11].

Dimensionless stress-time and velocity-time relationships of the graphical solution at positions $x=0$, $0.5 \lambda^{\prime} T, 1.0 \lambda^{\prime} T, 1.5 \lambda^{\prime} T$ are shown in figures 3 and 4 . Superimposed on this description are the numerical calculations for mesh spacings of $\Delta t=0.05 T, 0.0125 T$, and $0.00625 T$, and $\Delta x=\lambda \Delta t$. The numerical description is seen to converge to the more accurate graphical relationships as the mesh size is decreased. The symbols used to designate the numerical solution are made open and dark at alternate positions for clarity in the 3-dimensional plot of figure 3. Only during the plastic deformation at $x=1.0 \lambda^{\prime} T$ does the convergence of the numerical solution appear
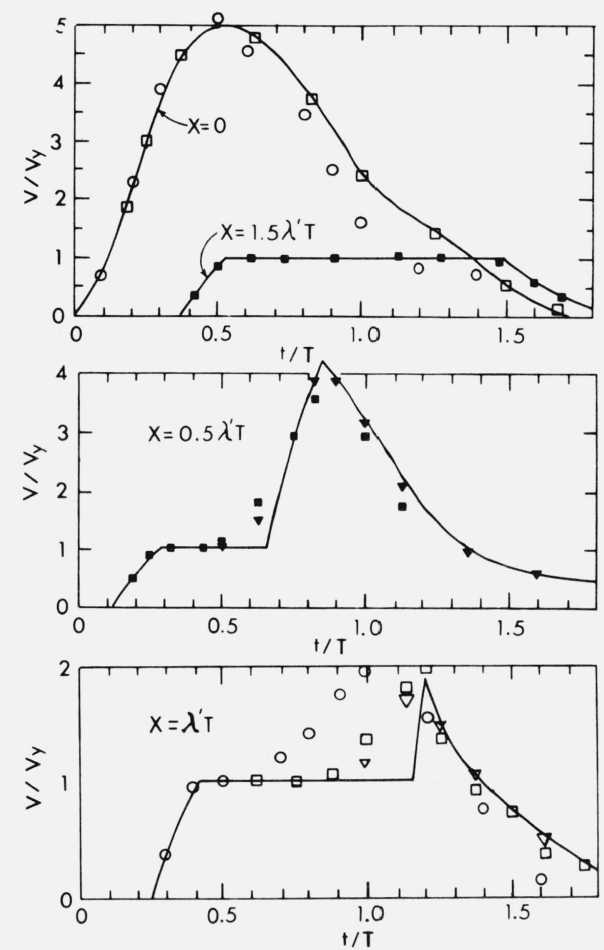

FIGURE 4. Graphical and numerical solution of stress pulse problem (dimensionless velocity-time plot). 
objectionably slow. Examination of the $x / \lambda^{\prime} T-t / T$ plane of figure 3 shows the width of the plastic zone, region III, at this point is the same order of magnitude as the smallest $\Delta x$ mesh spacing. Thus, the mesh size must be decreased severalfold to obtain a more accurate solution in this region. The location of the elastic-plastic boundaries exhibit similar behavior. That is, the numerical solution is reliable in locating the elastic-plastic boundaries of figure 3 to within the accuracy of the mesh size and the precision of the calculations.

An important observation is that the numerical calculations show no evidence of instability. This hypothesis is supported by the agreement between the graphical and numerical solutions in the region of constant state, on the unloading portion of the stresswave, and the totally elastic response at $x=1.5 \lambda^{\prime} T$. For the above and similar test problems, a theoretical energy balance of kinetic, elastic, and plastic energy to work input was found to be a measure of the overall accuracy of the numerical solution. This result is used in estimating the error in the numerical description of the following section where alternate methods of solution are not available.

\section{Constant-Velocity Impact of Tapered Rods}

Unlike the stress pulse problem of the previous section, the last integral term of eqs (5) and (6) is nonzero for a rod of nonuniform cross-sectional area. The additional terms cause no change in the numerical scheme illustrated in the flow diagram of figure 2 . Furthermore, since the difference relations of eqs (12) and (13) are consistent-that is, of the same degree of approximation-no complication in the observed behavior of the numerical solution is anticipated. Indeed, convergence of the numerical solution in this case was found to be similar if not faster than the results shown in figure 3 and other test problems. For example, the calculations presented in the following discussion for tapered roc's are, for the most part, convergent to 3 significarit figures for $\Delta t$ equal 0.254 and $0.127 \mu \mathrm{s}$. A test problem of a uniform, semiinfinite rod subjected to the same boundary conditions, stress-strain relationship, grid size, and total time of calculations also exhibited convergence to 3 significant figures, and at no time was the difference between the numerical and exact solutions greater than 4 percent. The theoretical energy balance also indicated that the accuracy of the solutions presented in this section is within this range. Although analytic or so-called closed form solutions of plastic-wave propagation in rods of nonuniform cross-sectional areas have received attention in the literature (see E. Wlodarczyk $[12,13]$ for a notable contribution and a list of important publications), the idealizations required in this approach are too restrictive for the interests of this investigation. In fact, while such descriptions employ a variety of analytical methods, the term closedform is sometimes misleading in that solutions often require numerical procedures and evaluation of

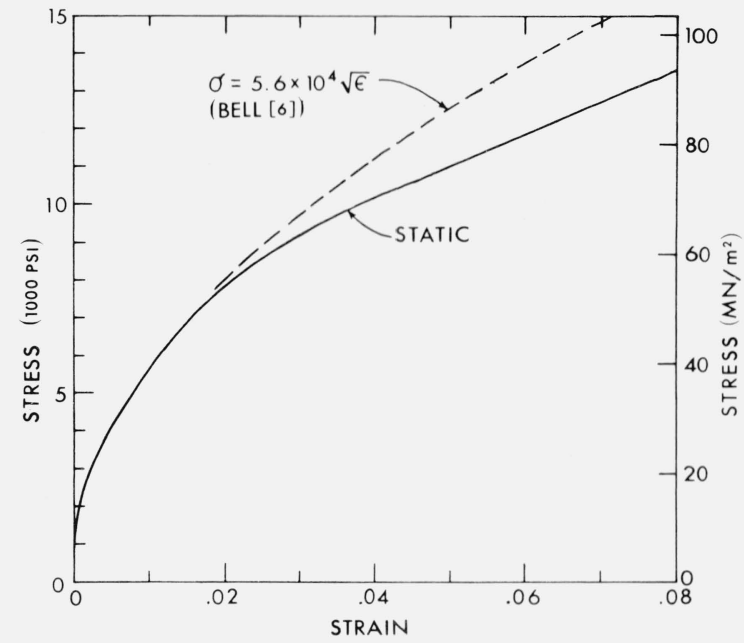

FIGURE 5. Stress-strain curves for commercially pure aluminum annealed $2 \mathrm{hr}$ at $1100^{\circ} \mathrm{F}\left(593^{\circ} \mathrm{C}\right)$ and furnace cooled.

implicit functions. Thus, more direct evaluation of the numerical method in this case was not attempted.

The material selected for investigation is commercially pure aluminum $(1100-0)$ which has been annealed for $2 \mathrm{hr}$ at $1100{ }^{\circ} \mathrm{F}\left(593^{\circ} \mathrm{C}\right)$ and furnace cooled. The stress-strain curve shown in figure 5 is obtained from the average data of 8 static tests, and is closely approximated by 20 linear segments for use in the computer code. Three specimen geometries presented for discussion and subsequently compared to experiments are a rod of uniform cross-sectional area and rods with a \pm 0.03 tapered slope. In each case, the rods are initially unstressed and 6 in $(152 \mathrm{~mm})$ in length; the boundary condition at the impact face corresponds to a constant-velocity impact except that the step in velocity has a finite rise-time. This phenomenon of longitudinal impact testing is caused by the physical limitation of achieving a plane impact of flat surfaces and is also influenced by the twodimensional wave structure of the deformation near the impact face. For an impact velocity of $800 \mathrm{in} / \mathrm{s}$ $(20 \mathrm{~m} / \mathrm{s})$, the loading condition was approximated by

$$
V=800 \sin \frac{\pi t}{80} \text { for } 0 \leqq t \leqq 40 \mu \mathrm{s}
$$

and

$$
V=800 \quad \text { for } t>40 \mu s
$$

The $40 \mu \mathrm{s}$ rise-time in the above relations was estimated from strain-time observations in the impact of uniform rods and agrees with similar data published by Bell [14]. From symmetry conditions of the longitudinal impact of two identical specimens, the constant-velocity loading, equal to one-half the speed of the projectile, can be shown to hold until the axial stress at the impact boundary is reduced to zero. 


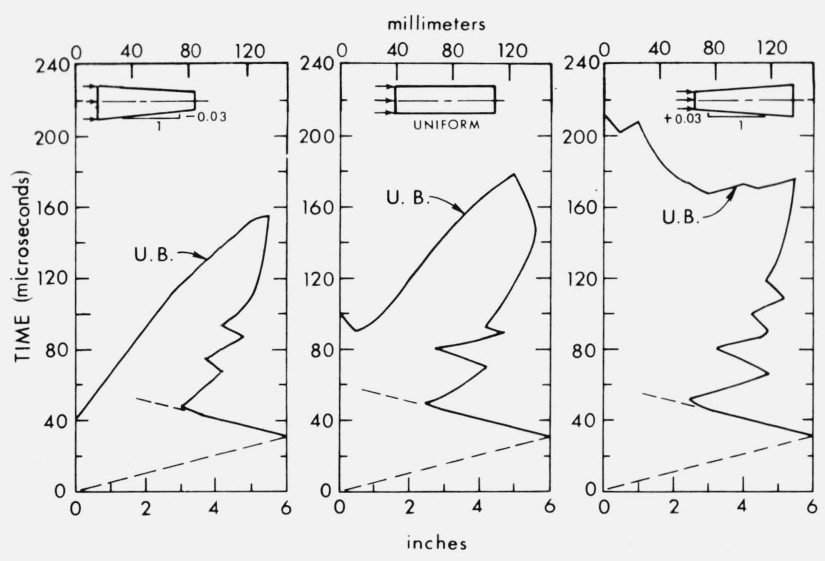

DISTANCE FROM IMPACT BOUNDARY

FigURE 6. Theoretical location of unloading boundary (U.B.) in $\mathrm{x}, \mathrm{t}$-plane.

It was hypothesized that the rise-time would be relatively constant for the uniform and tapered rods for the same impact velocity. At the free end of the rods, the stress is zero for all times.

The theoretical locations of the unloading boundaries in the $x, t$-plane for the above defined problems are shown in figure 6. In all cases, unloading is initiated at the free end, $x=6.0$ in $(152 \mathrm{~mm})$, and travels at the elastic speed into the solution field. Due to the interaction with the incident compressive wave, however, these disturbances are contained near the free end producing a sawtooth appearance. For the cross-sectional area decreasing in the direction of motion, -0.03 tapered rod, unloading also takes place at the impact face $(x=0)$ immediately after the velocity obtains a constant value ( $t$ greater than $40 \mu \mathrm{s})$. This boundary is seen to propagate at a near constant velocity of 0.188 times the elastic speed and produces a stress-pulse wave form. In the case of the uniform bar, a similar unloading pattern develops at a later time of $90 \mu \mathrm{s}$ and is attributed to the influence of the free end. For the cross-sectional area increasing in the direction of motion, +0.03 tapered rod, the eventual unloading along the bar starts near its center at a time of $160 \mu \mathrm{s}$.

The attenuation and amplification of the stress wave as a function of position along the bar and specimen geometry are shown in the diagrams of figure 7. Influencing the wave shape, in any one case, are the combined and interrelated effects of changes in mass along the bar and the occurrence of unloading. For example, while the cross-sectional area decreasing in the direction of motion tends to raise the transient stress level, the relatively rapid unloading of the specimen in this case limits maximum stress values to levels below the corresponding values in the uniform bar.

As indicated in the relationships of figure 7 , the stress can be expected to change from compression to tension. A tension stress can not obviously be
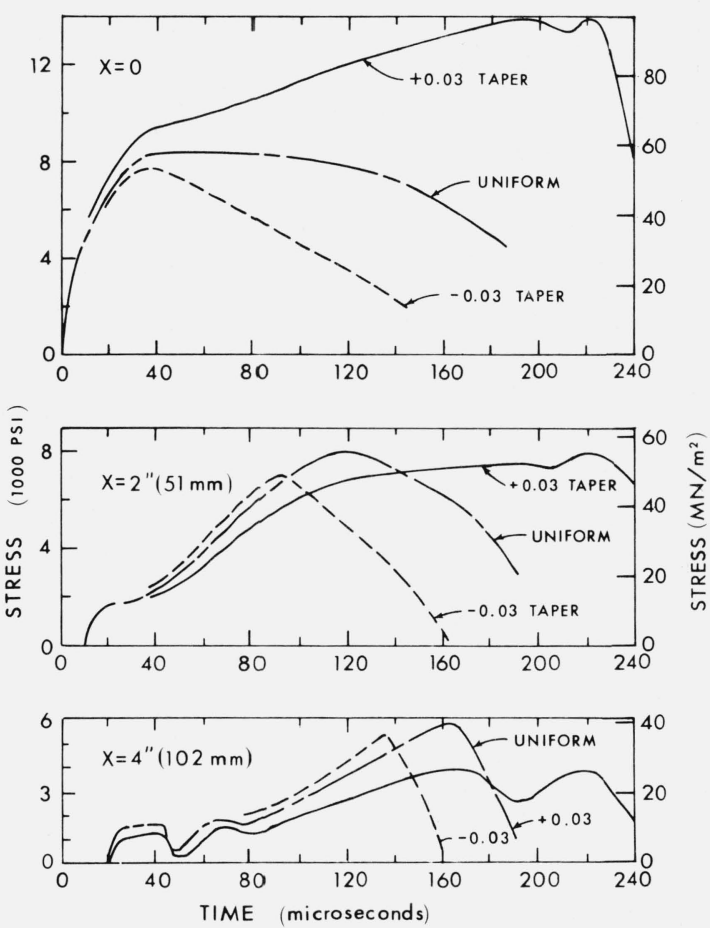

FIGURE 7. Theoretical stress-time relationships.

maintained at the impact faces, and the boundary condition at the moment when the stress changes sign should be changed from constant velocity to stress free. The solutions presented for discussion were terminated sometime after the specimen, had unloaded along its entire length. During this time, compression stresses were maintained at the impact face and the maximum tension stress in the bar only slightly exceeded 1200 psi $\left(8.27 \mathrm{MN} / \mathrm{m}^{2}\right)$, the virgin compression-yield stress of the material. Nonetheless, this behavior points to the need of incorporating the response of the material on stress reversal, e.g., a Bauschinger effect, into the dynamic analysis. Also, there is no guarantee that plastic deformation cannot reappear at a later time. The relative importance of these uncertainties in the present problems will be determined from experimental observations.

\section{Experimental Observations}

A constant-velocity loading is achieved by the axial collision of identical specimens using a $3 / 4$-in $(19 \mathrm{~mm})$ bore gas gun. A schematic view of ihe experimental setup is shown in figure 8. Fibrous washers are used to make the tapered projectiles concentric with the barrel. Impact velocities are measured by a light screen and photomultiplier tube system whose signals alternately start and stop a timer on passage of the projectile. The instrumented specimens are axially alined with the barrel and rest on point supports. 


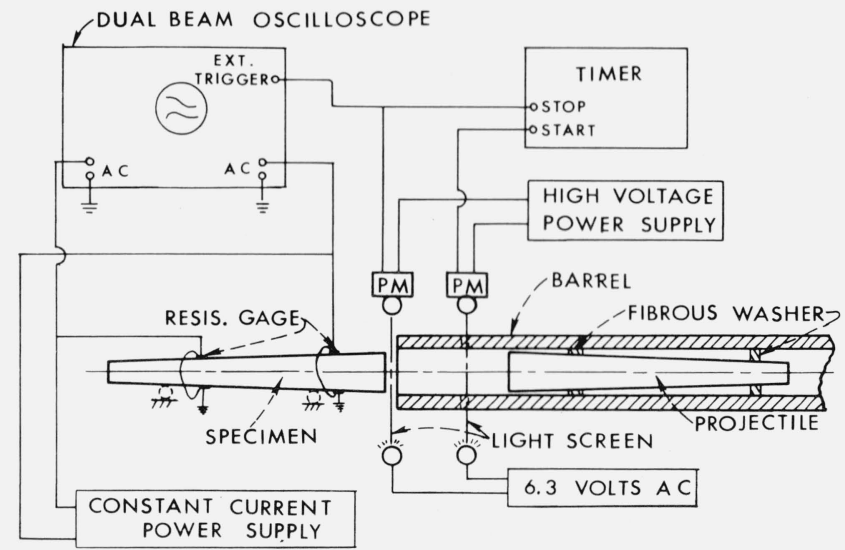

FIGURE 8. Schematic diagram of experimental apparatus and instrumentation.

Except for the small constraint of the fibrous washers, the projectile and specimen are in free flight during collision.

Strain-time profiles are measured at cross-sectional distances of 1,2 , and 4 in $(25,51$, and $102 \mathrm{~mm})$ from the impact face with epoxy back, 0.063-in $(1.6 \mathrm{~mm})$ gage length, constantan, resistance gages. For each measurement, two gages are mounted diametrically opposite one another with either RTC epoxy or Eastman 910 cements; the gages are wired in series and connected to a constant current power supply of $25 \mathrm{~mA}$. Before each test, the circuit is calibrated by shunting a series of resistors across the gages. Outputs from two sets of gages are fed to a-c coupled, vertical amplifiers of a dual beam oscilloscope. The horizontal sweep of the oscilloscope beams is triggered by the signal from the photomultiplier tube just before impact. The writing speed is $20 \mu \mathrm{s} / \mathrm{cm}$, and the vertical sensitivity is between 20 and $100 \mathrm{mV} / \mathrm{cm}$ depending on the maximum strain level. The response of the oscilloscope for the latter operating condition, better than $1 \mu$ s rise-time, is more than adequate for the observed transient signals. The signals are recorded by an open shutter camera.

Both the experimental and theoretical strain-time profiles for the three problems are shown in figures 9, 10 , and 11. The designated points on the experimental traces correspond to calibration levels. Asterisks denote interpolations between calibration points. The reproducibility of the data is considered good. Two important considerations in obtaining representative data are (1) to minimize bending deformation caused by unsymmetrical and skew impact of specimen and projectile, and (2) to accommodate the one-dimensional approximation to the motion by making measurements in that section of the specimen where radial inertia effects are negligible. To investigate these factors in the present experiments, tests were made in which two sets of gages are mounted $90^{\circ}$ apart at the same cross-sectional distance. One set of gages is mounted in the standard way to measure the axial strain; the other set of gages, 0.25 in $(6.4 \mathrm{~mm})$ length by 0.032 in $(0.8 \mathrm{~mm})$ width, is mounted so as to measure circumferential strain. Typical data is shown in figure 10, test No. 14 for the uniform rod at the one inch position. The axial strain is seen to equal twice the circumferential strain. These results are in agreement with the incompressible behavior of plastic flow for a uniaxial stress state; and therefore, indicate that effects of bending and radial motion are small.

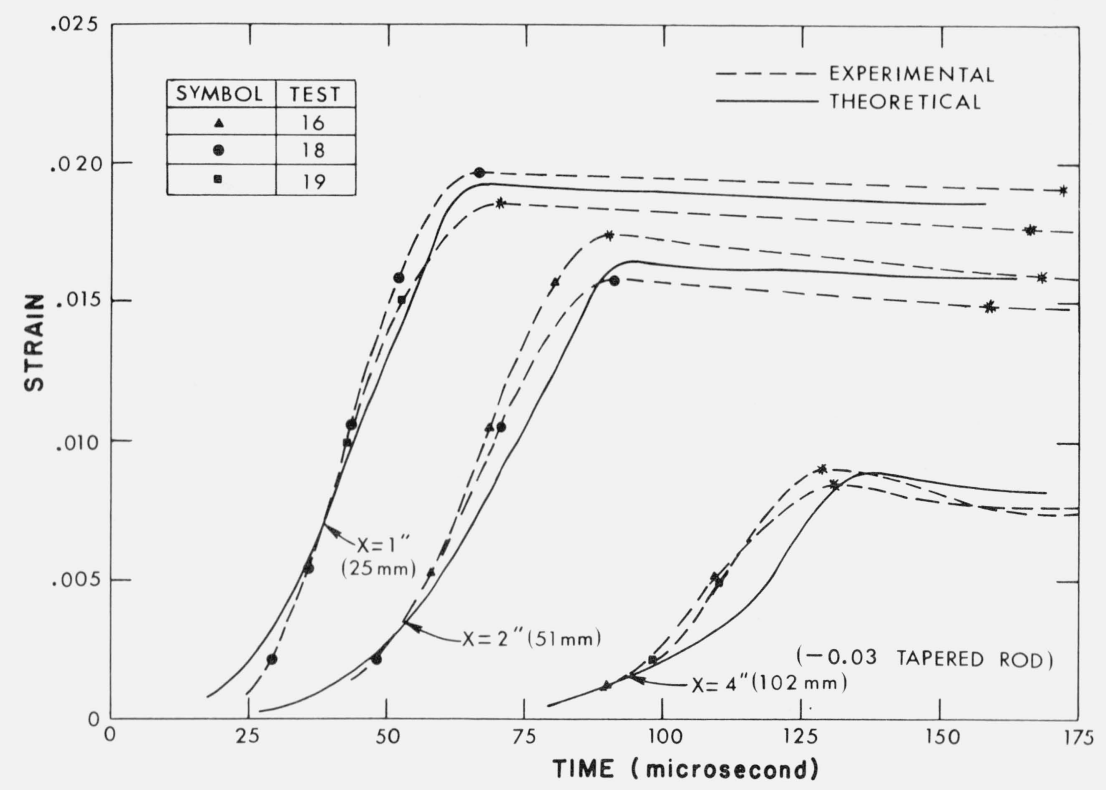

Figure 9. Experimental and theoretical, axial strain-time profiles (-0.03 tapered rod). 


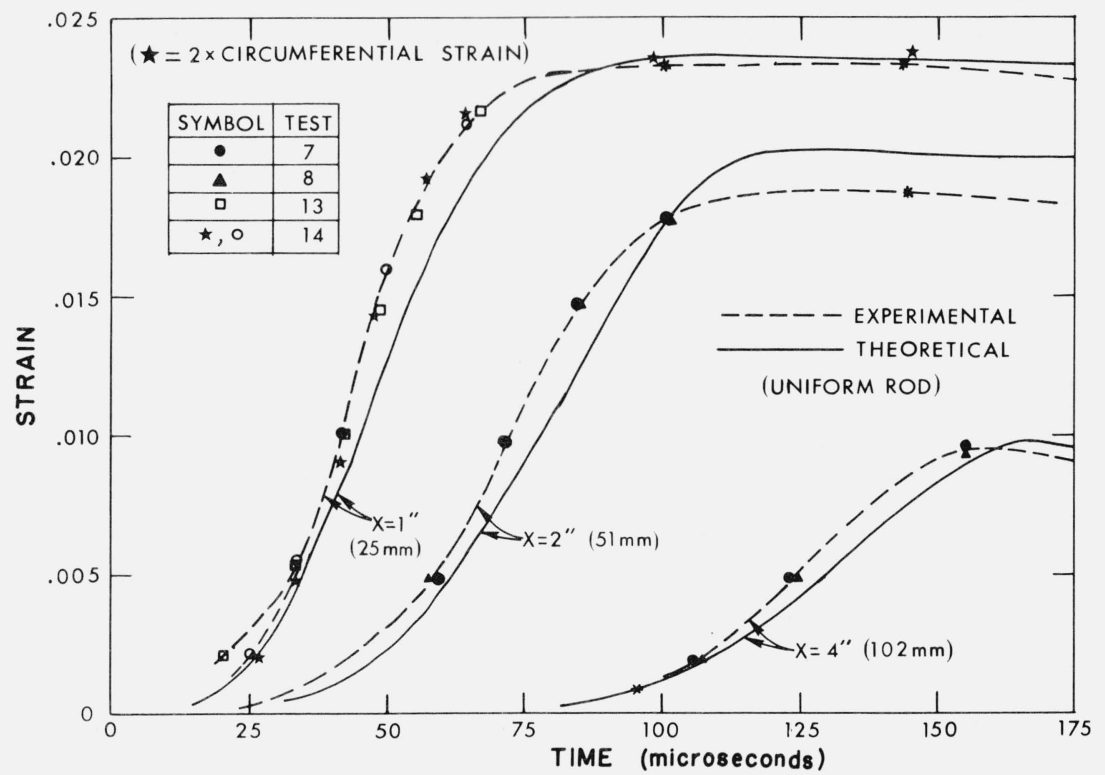

FIGURE 10. Experimental and theoretical, axial strain-time profiles (uniform rod).

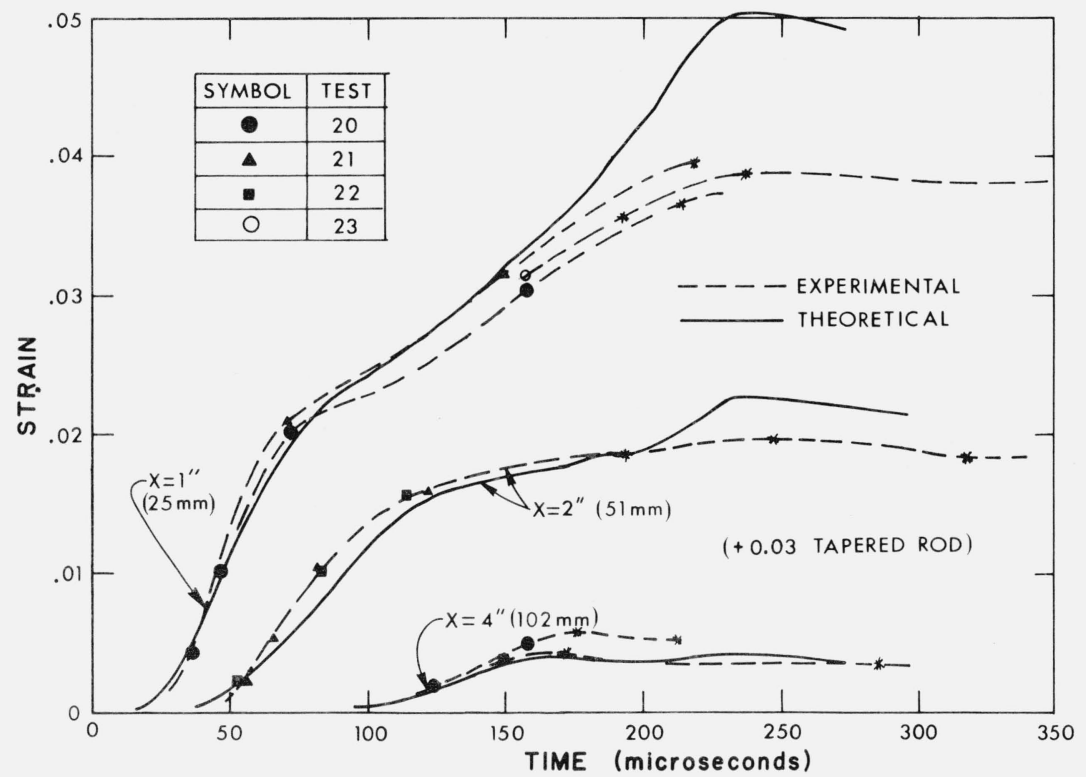

FIGURE 11. Experimental and theoretical, axial strain-time profiles +0.03 tapered rod).

Considering the approximation of the boundary condition defined in eq (15), comparison of the theoretical and experimental strain-time profiles show the theory and method of solution to be an accurate model of the deformation. That is, the general features of the strain waves predicted by the numerical solution are, for the most part, in quantitative agreement with the observed behavior. Of particular note is the development of the unloading boundary in the -0.03 tapered rod which is well defined by the peak levels of the strain-time traces. This boundary is seen not only to initiate near the impact face but its propagation speed is within 10 percent of the theoretical value.

At 1 in from the impact end, each of the three specimens experienced a near constant strain-rate of $40 / \mathrm{s}$. For the uniform and -0.03 tapered rods, the calculated maximum strain levels of 0.024 and 0.019 , respectively, are in close agreement with experimental values. In the case of +0.03 tapered rod, however, the measured maximum strain is 

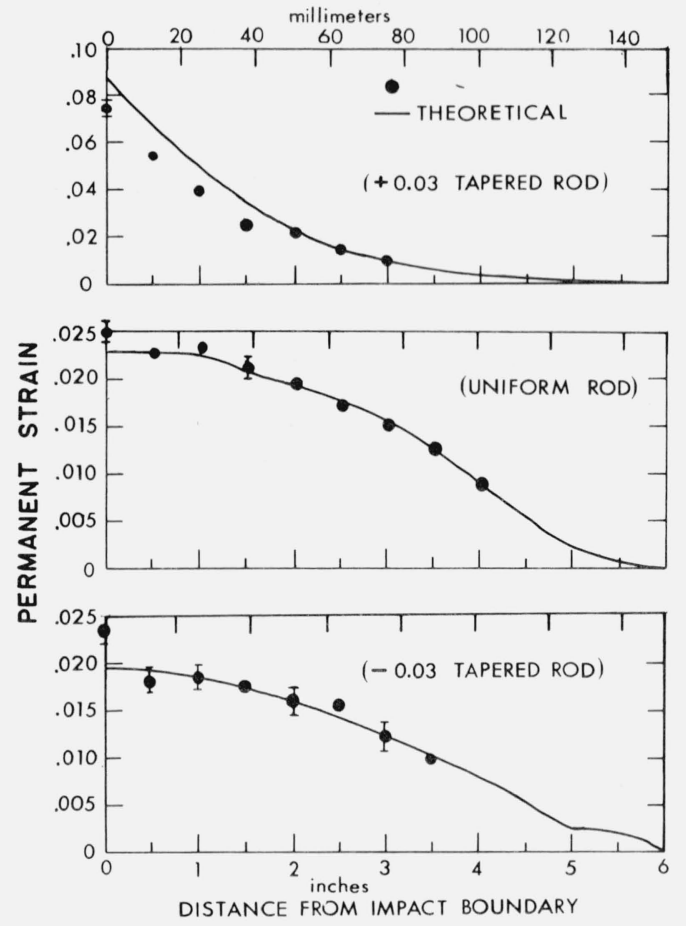

FigurE 12. Experimental $(2 \times$ diametral strain $)$ and theoretical, axial, permanent-strain distribution.

significantly less than the predicted value. While this difference suggests a strain-rate dependence of material response, the above results can also be rationalized in light of the dynamic stress-strain curve of this material reported by Bell [6]. As shown in figure 5, Bell's dynamic curve diverges from the static relationship for strains above 0.02 . Furthermore, at a stress level of $11 \mathrm{ksi}\left(75.8 \mathrm{MN} / \mathrm{m}^{2}\right)$, the difference in strain between the dynamic and static curve is the same order of magnitude observed in figure 11.

Post-mortem examinations of the specimens and projectiles are made by measuring the diametral deformation with micrometers of 0.0001 in resolution. As shown in figure 12, twice the diametral strain is found nearly equal to theoretical prediction of the permanent, axial-strain distribution except for higher values of strain noted above. These results also satisfy the incompressible behavior of plastic flow, and resolve an earlier contention that plastic deformation of the longitudinal impact problems is confined to the time periods described in figures 9,10 and 11 . Thus, the restriction of the stress-strain curve to linear-elastic response on stress reversal is of little consequence in this study. It is noted that this restriction on the stress-strain curve is caused by the absence of a unique formulation of plastic flow on cyclic loading, and is not due to any limitation in the method of solution. Comparison of data in figures 11 and 12 also shows the resistance gages are reliable in measuring the maximum strain of these magnitudes at the 1 -in $(25-\mathrm{mm})$ position.
The adequacy of the rate-independent theory to describe the dynamic deformation of annealed aluminum reported above is in general concordance with the recent studies of Bodner and Clifton [15] on the stress-pulse propagation in uniform rods at room temperature; and of Schultz, Tuschak, and Vicario [16] on the transverse wire impact measurements. It would seem unlikely that close correlation between theory and experiment would be obtained for such diverse studies if the governing stress-strain curve were ratedependent to the degree reported in references 7 and 8 . In the latter references, the rate-dependent behavior of 0-tempered, commercially pure aluminum is determined by a split Hopkinson bar for a strain rate of $40 / \mathrm{s}$. These data show a marked increase in flow stress above the static curve for strains between yield point and 0.10 . Since the theoretical-experimental comparisons cited above do not measure simultaneously stress and strain at a material point these results are a necessary but not a sufficient measure of materials' rate-independent behavior. Nonetheless, results of the split Hopkinson bar suffers from similar limitations in assuming the stress state is quasi-static and uniaxial; and therefore, the seemingly contradictory results are noteworthy.

\section{Conclusion}

An iterative-explicit, numerical solution has been formulated to a large class of problems in the onedimensional, rate-independent theory of plastic-wave propagation. Convergence and stability behavior of the method are found sufficient for machine computation. For example, solutions presented in this study with $\Delta t=0.254 \mu \mathrm{s}$ required approximately $5 \mathrm{~min} / 1000$ time steps on a Univac 1108 computer. The solution has been used to design experiments in the longitudinal impact of uniform and tapered rods of annealed aluminum. Close correlation between theory and measured response has been obtained for strainrates and strains as high as $40 / \mathrm{s}$ and 0.024 . The onedimensional theory is, however, of questionable value near the impact face, within one-half diameter, where the wave structure is two-dimensional. Also consistent with the uniaxial approximation is the requirement that the change in cross-sectional area be kept to some minimum, nominal value.

\section{References}

[1] von Kármán, Th., On the Propagation of Plastic Deformation in Solids, NDRC Report No. A-29, OSRD No. 365 (1942).

[2] Taylor, G. I., The Plastic Wave in a Wire Extended by an Impact Load, The Scientific Papers of G. I. Taylor (Edited by G. K. Batchelcr, University Press, Cambridge) 467-479 (1958).

[3] Rakhmatulin, Kh. A., The Propagation of unloading waves, Prikladnaya Matematika i Mekhanika 9, 1 (1945).

[4] Lee, E. H., A boundary value problem in the theory of plastic wave propagation. Quarterly of Applied Mathematics 10, 335-346 (1952).

[5] Bell, J. F., The Dynamic Plasticity of Metals of High Strain Rates, Behavior of Materials Under Dynamic Loading (Ed. N. J. Huffinton, A SME, N.Y.) 19-41 (1965). 
[6] Bell, J. F., Experincental study of the interrelation between the theory of dislorations in polycrystalline media and finite wave propagation in solids, J. Applied Physics 32, 10, 1982-1993 (Oct. 1961).

[7] Holt, D. L., Babcock, S. G., Green, S. L., and Maiden, C. J., The strain-rate dependence of the flow stress in some aluminum alloys, Trans. American Society for Metals 60, 2, 152160 (June 1967).

[8] Lindholm, U. S., and Yeakley, L. M., High strain-rate testing: tension and compression, Experimental Mechanics 8, 1-9 (Jan. 1968).

[9] Forsythe G. E., and Wasow, W. R., Finite-Difference Methods for Partial Differential Equations, ch. 2 (John Wiley \& Sons, New York, N. Y. (1960).

[10] Rakhmatulin, Kh. A., and Den' Yanov, Yu. A., Strength Under High Transient Loads, Israel Program for Scientific Translations, Jerusaleum (Daniel Davey and Co., Inc., New York, N.Y.) $31-33$ (1966).

[11] Shapiro, G. S., Prodol' nye kolebaniya sterzhnei (Longitudinal vibrations of bars), Prikladnaya Matematika i Mekhanika 10, 5 and 6 (1946).

[12] Wlodarczyk, E., Propagation of elastic-plastic and shock waves in a bar of finite length and monotone decreasing cross-sectional area, Proceeding of Vibration Problems, Warsaw 2, 7, 135-153 (1966).

[13] Wlodarczyk, E., Propagation of a loading and unloading wave in a bar with monotone increasing cross-sectional area and curvelinear $(\sigma-\mathrm{E})$ relation, Bulletin De L'Academie, Polonaise Des Sciences, Serie de science techniques 14,3, 185-252 (1966).

[14] Bell, J. F., Study of Initial Conditions in Constant Velocity Impact, J. Applied Physics 31, 12, 2188-2195 (Dec. 1960).

[15] Bodner, S. R., and Clifton, R. J., An experimental investigation of elastic-plastic pulse propagation in aluminum rods, J. Applied Mechanics 34, 1 (Mar. 1967).

[16] Schultz, A. B., Tuschak, P. A., and Vicario, A. A., Jr., Experimental evaluation of material behavior in a wire under transverse impact, J. Applied Mechanics 34, 2 (June 1967).

\section{Appendix: Transformation of Governing Equations into Normal Form}

Adopting a Lagrangian description of the deformation, let $u(x, t)$ denote the particle displacement from its initial position $x$ in an unstrained rod of nonuniform cross-sectional area. Hence, the nominal strain, change in length per unit initial length, is $\epsilon=\frac{\partial u}{\partial x}$, and the particle velocity is $v=\frac{\partial u}{\partial t}$. The kinematic identity follows

$$
\frac{\partial \epsilon}{\partial t}=\frac{\partial v}{\partial x}
$$

and the equation of motion is

$$
\frac{\partial \sigma}{\partial x}+\rho \frac{\partial v}{\partial t}+\frac{\partial A}{\partial x} \frac{\sigma}{A}=0
$$

where $\sigma$ denotes the nominal stress, force per unit area, $\rho$ is the mass density, and $A$ is the cross-sectional area. A determinate system of equations is obtained by introducing the stress-strain relation, $\sigma=f(\epsilon)$, of the material in the form

$$
\frac{\partial \sigma}{\partial t}-f^{\prime} \frac{\partial \epsilon}{\partial t}=0
$$

where $f^{\prime}=d \sigma / d \epsilon$. In the above formulation compressive stresses and strains are positive, and conservation of mass is implicitly satisfied.

The three governing equations can be written in the matrix form

$$
L[\bar{w}]=A \bar{w}_{x}+B \bar{w}_{t}+d \bar{w}=0
$$

where $\bar{w}=(v, \sigma, \epsilon)$ and

$$
A=\left[\begin{array}{ccc}
0 & 1 & 0 \\
1 & 0 & 0 \\
0 & 0 & 0
\end{array}\right] ; B=\left[\begin{array}{ccc}
\rho & 0 & 0 \\
0 & 0 & 1 \\
0 & 1 & -f^{\prime}
\end{array}\right] ; d=\left[\begin{array}{ccc}
0 & \frac{\partial A}{\partial x} \frac{1}{A} & 0 \\
0 & 0 & 0 \\
0 & 0 & 0
\end{array}\right]
$$

The characteristic roots $\lambda$ of the above equations are obtained by setting the determinate of the characteristic matrix $\Lambda$ to zero, that is,

$$
\operatorname{det} \Lambda=\operatorname{det}(A-\lambda B)=0
$$

and are found to be

$$
\lambda= \pm\left(f^{\prime}(\epsilon) / \rho\right)^{1 / 2} ; 0 .
$$

The nonzero roots are recognized as the wave speed corresponding to a strain $\epsilon$.

Associated with the characteristic matrix and each root is a null vector $\bar{l}$ which satisfies

$$
\Lambda \bar{l}=0 .
$$

For eqs (4a), the null vectors are to an arbitrary scale factor

$$
\begin{aligned}
& \bar{l}_{+}=\left(\lambda, \lambda^{2} \rho, 1\right) \quad \text { when } \lambda=+\left(f^{\prime} / \rho\right)^{1 / 2} \\
& \bar{l}_{-}=\left(\lambda,-\lambda^{2} \rho,-1\right) \quad \text { when } \lambda=-\left(f^{\prime} / \rho\right)^{1 / 2} \\
& \bar{l}_{0}=(0,0,1) \quad \text { when } \lambda=0 .
\end{aligned}
$$

The normal form of eq (4a) is obtained by their scalar product with the null vector, that is

$$
\bar{l} \cdot L[\bar{w}]=0 .
$$

Performing the above operation one obtains

$$
\lambda\left(\frac{\partial \sigma}{\partial x}+\rho \frac{\partial v}{\partial t}+\frac{\partial A}{\partial x} \frac{\sigma}{A}\right)+\lambda^{2} \rho\left(\frac{\partial \epsilon}{\partial t}+\frac{\partial v}{\partial x}\right)+\left(\frac{\partial \sigma}{\partial t}-f^{\prime} \frac{\partial \epsilon}{\partial t}\right)=0
$$

$\lambda\left(\frac{\partial \sigma}{\partial x}+\rho \frac{\partial v}{\partial t}+\frac{\partial A}{\partial x} \frac{\sigma}{A}\right)-\lambda^{2} \rho\left(\frac{\partial \epsilon}{\partial t}+\frac{\partial v}{\partial x}\right)-\left(\frac{\partial \sigma}{\partial t}-f^{\prime} \frac{\partial \epsilon}{\partial t}\right)=0$

$$
\left(\frac{\partial \sigma}{\partial t}-f^{\prime} \frac{\partial \epsilon}{\partial t}\right)=0
$$


Substituting eq (3) and the last of the above equations into the first two and rearranging terms, the normal form is $\left(\frac{\partial \sigma}{\partial t}-\lambda \frac{\partial \sigma}{\partial x}\right)-\lambda \rho\left(\frac{\partial v}{\partial t}-\lambda \frac{\partial v}{\partial x}\right)-\lambda \frac{\partial A}{\partial x} \frac{\sigma}{A}=0$.

(11a)

$$
\left(\frac{\partial \sigma}{\partial t}+\lambda \frac{\partial \sigma}{\partial x}\right)+\lambda \rho\left(\frac{\partial v}{\partial t}+\lambda \frac{\partial v}{\partial x}\right)+\lambda \frac{\partial A}{\partial x} \frac{\sigma}{A}=0
$$

(Paper 72C4-280) 